\title{
RETRACTED ARTICLE: Analysis of serum levels and tissue expression of galectin-1 and galectin-3 as noninvasive biomarkers in osteosarcoma patients
}

\author{
Ali Akbar Esmailiejah ${ }^{1}$ • Afshin Taheriazam ${ }^{2}$ - Mohammad Reza Golbakhsh ${ }^{3}$. \\ Mohammad Jamshidi $^{4,5}$ - Mohammadreza Shakeri ${ }^{6}$ Emad Yahaghi ${ }^{7}$. \\ Mehdi Moghtadaei ${ }^{8}$
}

Published online: 9 October 2015

(C) International Society of Oncology and BioMarkers (ISOBM) 2016

\section{RETRACTED ARTICLE: Tumor Biol.}

DOI 10.1007/s13277-015-4194-4

This article has been retracted at the request of the Editor-in-Chief, the International Society of Oncology and BioMarkers (ISOBM) and the Publisher per the Committee on Publication Ethics guidelines. The article shows evidence of irregularities in authorship and in the materials and methods during the submission process, there is strong reason to believe that the peer review process was compromised and

Electronic supplementary material The online version of this article (doi:10.1007/s13277-015-4194-4.) contains supplementary material, which is available to authorized users.

Mehdi Moghtadaei

mmoghtadaei@gmail.com

1 Department of Orthopedics, Akhtar Hospital, Shahid Beheshti University of Medical Sciences, Tehran, Iran

2 Department of Orthopedics, Tehran Medical Sciences Branch, Islamic Azad University, Tehran, Iran

3 Department of Orthopedics, Sina Hospital, Tehran University of Medical Sciences, Tehran, Iran

4 Cellular and Molecular Research Center, Zahedan University of Medical Sciences, Zahedan, Iran

5 Department of Clinical Biochemistry, School of Medicine, Zahedan University of Medical Sciences, Zahedan, Iran

6 Department of Orthopaedic and Trauma Surgery, Birjand University of Medical Sciences, Birjand, Iran

7 Department of Molecular Biology, Baqiyatallah University of Medical Sciences, Tehran, Iran

8 Department of Orthopedic, Rasoul-e-Akram Hospital, Iran University of Medical Science, Tehran, Iran the article contains paraphrased content from the following article:

Xuesong Zhoua, Jie Jinga, Jing Pengc, Wen Maoa, Yizhou Zhenga, Du Wanga, Xin Wanga, Zhiming Liua, Xiangming Zhanga, Expression and clinical significance of galectin-3 in osteosarcoma, Gene, (2014) 546:2 403-407 http://dx.doi.org/ 10.1016/j.gene.2014.04.066

As such the validity of the content of this article cannot be verified.

The online version of this article contains the full text of the retracted article as electronic supplementary material. 